\title{
Actively actuated all dispenser printed thermochromic smart fabric device
}

Z. Ahmed, Y.Wei, R.Torah and J.Tudor

This paper for the first time reports an all printed actively actuated thermochromic smart fabric device using a state of the art dispenser printing technique. The device consists of a UV curable thermochromic ink with an activation temperature of $33^{\circ} \mathrm{C}$ actuated by conductive track based printed heater. The device is printed on untreated polyester cotton fabric. It offers a significant improvement in flexibility and design freedom over the state of the art thermochromic fabric devices. The printed device changes colour from black to green in $10.8 \mathrm{~s}$ using $1.46 \mathrm{~W}$ DC power. It is shown that the time required for the device to change colour reduces tenfold with only a threefold increase in input power. It can be fabricated on other fabric or flexible substrates and in a range of colours and activation temperatures depending on the formulation chosen. The printed device is 30 $x 21.5 \mathrm{~mm}$ which can be scaled up or down to suit the application. It can be used as an indicator in combination with sensors for smart fabric applications.

Introduction: Smart fabrics can sense and react to various stimuli with functionalities such as communication, power transmission and information processing [1]. They can be realised by combining fabrics with smart and functional materials such as electroluminescent, piezoelectric and conductive. Colour change functionality can be incorporated in the fabrics by combining them with thermochromic (TC) materials. TC materials change colour in response to a temperature change.

Several artists and designers have used these materials with fabrics to integrate functional and aesthetic qualities. The colour change property in TC fabrics can be actively controlled by controlling temperature to produce desired colour/pattern changes. Shimmering flower [2], ambikraf [3], fabrication bag [4] and TC information surfaces [5] are some examples of colour changing fabrics. Applications of TC fabrics include non-emissive displays, interior design, art and as indicators in interactive systems.

This paper reports a novel all printed approach for fabricating an actively actuated TC fabric device. The dispenser printed device overcomes existing limitations of design freedom, inflexibility and the requirement to use of multiple fabrication techniques. Dispenser printing is a new technique offering a direct write capability, obviating the need to use design specific screens for each design as required in screen printing. Using a printed heater in this TC device addresses current limitations in the state of the art by offering a flexible heating mechanism integrated in the fabric.

Background: Active actuation of a TC fabric is the capability to bring about a colour change in TC materials when desired. Fabrication of actively actuated TC fabrics is a two-step process consisting of the application of the TC material on fabric and the integration of a heater for temperature, and thus transition, control. Previously, the two steps have been performed using distinct fabrication techniques.

TC materials have been applied on the fabric using screen printing which requires bespoke screens to print each specific design. State of the art heating mechanisms for TC fabrics are conductive yarn technology [2, 4], PCB based heaters [5] and Peltier semiconductors [3].Conductive yarns need to be woven or stitched which limits the design freedom as the heater path follows the physical location of these yarns in a fabric substrate. PCB based heaters and Peltier semiconductors are inflexible and require the TC fabric to maintain contact with them.

Fabrication Technology: Dispenser printing is a new direct-write method where a material is additively deposited on a substrate in a computer defined pattern. It is a novel state of the art process developed for use in printed smart fabrics by the University of Southampton [6]. It offers features of custom patterning, rapid prototyping and the ability to print multi-layered and multi-material structures.

Dispenser technology uses pressure to dispense a specific quantity of material on a substrate. This work uses a pneumatic dispenser which connects to a syringe containing the ink, and linear XYZ movement stages all connected to a PC to control the position, speed, applied pressure, resolution and dispense time. The current printer, developed at the University of Southampton, offers a printing area of $25 \mathrm{~cm}^{2}$ and a printing resolution of $2 \mu \mathrm{m}$. The printer can fill a designated print area as droplets where an ink is deposited on a substrate in the form of successive droplets or as filaments where the ink is deposited on the substrate as adjacent straight lines. In both cases, with the correct settings, once the ink is deposited it coalesces with nearby ink to form a homogenous layer. 
Design of an All Printed TC Fabric Device: The all printed TC fabric device consists of three layers: the fabric substrate, the TC layer and a heater layer.

- Fabric substrate: The fabric may offer a smooth or rough surface for printing depending on the type of fabric used.

- Heater layer: Consists of printed conductive tracks in a specific pattern to provide the desired heat profile.

- TC layer: Consists of a printed leuco dye based TC ink which can be formulated to change from one colour to another colour upon actuation. The ink can be formulated to change colour at any temperature between $-150^{\circ} \mathrm{C}$ and $150^{\circ} \mathrm{C}[7]$ called the activation temperature.

Figure 1 below shows the layout of the three layers in the TC fabric device reported in this work.

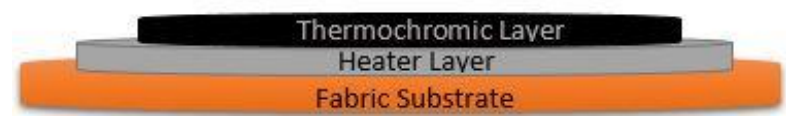

Fig. 1 Design layout of TC fabric device

Materials Used for Fabrication of TC Fabric Device: The TC device is fabricated on untreated woven 65/35 polyester cotton blend. Polyester cotton, a commonly used fabric, offers a challenging surface for printing due to high porosity and high surface roughness. High variation within the polyester cotton surface leads to non-uniform printing of silver layers which adversely affects their electrical properties and durability. An interface layer can be dispenser printed on the polyester cotton fabric to significantly reduce its surface roughness [6].

Electra EFV4/4965 dielectric, a UV curable ink is used for printing interface on the polyester cotton fabric in this work. Figure 2 shows the 2-D surface profiles of polyester cotton fabric and Electra EFV4/4965 printed on the polyester cotton fabric.

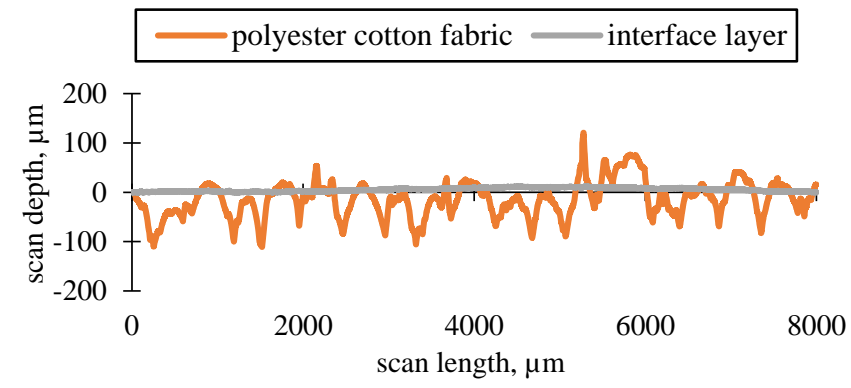

Fig. 2 Surface profile of polyester cotton with and without a Electra EFV4/4965 interface layer

Fabinks- C4001 silver ink is used for printing the heater in the TC device. It is a heat curable ink with a resistivity of $\leq$ $3.81 \mathrm{E}-07 \Omega . \mathrm{m}$, it is cured at $120^{\circ} \mathrm{C}$ for $8-10$ minutes.

Fabinks-UV-TC0233 ink is used for incorporating colour change function on the fabric. It is a UV curable ink which reversibly changes colour from black to green with an activation temperature of $33^{\circ} \mathrm{C}$. The UV curable inks are cured, after printing, using a Panacol-Elosol UV-P 280 ultraviolet point source. The prints were exposed to $2000 \mathrm{~mW} / \mathrm{cm}^{2} \mathrm{UV}$ radiation for 60 seconds.

Fabrication of Actively Actuated TC Device on Polyester Cotton: The TC device is printed in the following three steps.

- Firstly, a $38 \times 30 \times 0.237 \mathrm{~mm}$ interface is printed on the fabric to provide a smooth surface for subsequent heater layer. The interface is printed in the form of filaments.

- A $30 \times 21 \times 0.026 \mathrm{~mm}$ meander line pattern of conductive tracks is printed on the interface layer next. The heater pattern produced a resistance of $2.28 \Omega$ It is printed as a series of droplets of silver ink.

- Lastly, $28 \times 21.5 \times 0.187 \mathrm{~mm}$ TC layer is printed on the top of the heater layer to cover it. It is printed as adjacent filaments.

Figure 3 shows the interface layer, printed heater and final TC device before and after a colour change. Table 1 below shows dispenser printer settings for printing the three inks used for fabrication. The print settings for each ink were identified using similar optimisation process as our previous work [6]. The settings are predominantly influenced by the ink rheology and surface properties of the substrate. 


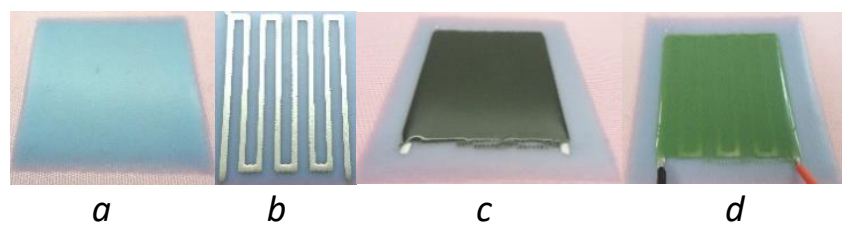

Fig. 3 Printed (a) interface layer on polyester cotton fabric (b) heater (c) final TC device before colour change (d) after colour change

Table 1 Dispenser printer settings for printing the TC device

\begin{tabular}{|c|c|c|c|c|c|c|c|}
\hline \multicolumn{8}{|c|}{ Interface ink settings } \\
\hline $\begin{array}{c}\text { Pressure } \\
(\mathrm{kPa})\end{array}$ & \multicolumn{2}{|c|}{ Vacuum (kPa) } & \multicolumn{2}{|c|}{$\begin{array}{c}\text { Nozzle } \\
\text { height }(\mu \mathrm{m})\end{array}$} & \multicolumn{2}{|c|}{$\begin{array}{c}\text { Y- resolution } \\
(\mathrm{mm})\end{array}$} & Speed $(\mathrm{mm} / \mathrm{s})$ \\
\hline 20.0 & \multicolumn{2}{|c|}{1.5} & & 200 & \multicolumn{2}{|c|}{0.20} & 5 \\
\hline \multicolumn{8}{|c|}{ Silver ink settings } \\
\hline $\begin{array}{c}\text { Pressure } \\
(\mathrm{kPa})\end{array}$ & $\begin{array}{l}\text { Dispense } \\
\text { time (ms) }\end{array}$ & $\begin{array}{l}\text { Vac. } \\
\text { (kPa) }\end{array}$ & \multicolumn{2}{|c|}{$\begin{array}{c}\text { Nozzle } \\
\text { height }(\mu \mathrm{m})\end{array}$} & $\begin{array}{l}\text { X-res. } \\
(\mathrm{mm})\end{array}$ & $\begin{array}{l}\text { Y- res. } \\
(\mathrm{mm})\end{array}$ & $\begin{array}{l}\text { Speed } \\
(\mathrm{mm} / \mathrm{s})\end{array}$ \\
\hline 52.5 & 11 & 1.5 & & 150 & 0.50 & 0.40 & 1 \\
\hline \multicolumn{8}{|c|}{ TC ink settings } \\
\hline $\begin{array}{c}\text { Pressure } \\
(\mathrm{kPa})\end{array}$ & $\begin{array}{l}\text { Vacuum } \\
\text { (kPa) }\end{array}$ & \multicolumn{2}{|c|}{$\begin{array}{c}\text { Nozzle } \\
\text { height }(\mu \mathrm{m})\end{array}$} & \multicolumn{2}{|c|}{$\mathrm{Y}$ - resolution $(\mathrm{mm})$} & \multicolumn{2}{|c|}{ Speed $(\mathrm{mm} / \mathrm{s})$} \\
\hline 40.0 & 1.0 & \multicolumn{2}{|c|}{150} & \multicolumn{2}{|c|}{0.26} & \multicolumn{2}{|r|}{5} \\
\hline
\end{tabular}

Actuation of TC Device: The fabricated TC device can be actuated by supplying power to the contact pads of the heater. Current passing through the tracks produces resistive heating which increases the temperature of the device above $33^{\circ} \mathrm{C}$ causing it's colour to change from black to green. When the power supply to the heater is cut off, it loses the heat to the environment reversing the colour change.

Actuation response rate is a key factor that influences applications of TC fabric devices. Actuation response rate of the fabricated TC device is dependent on ambient temperature, substrate material and input power. The relationship between input power and actuation response rate of the device was studied by varying the magnitude of the driving current from $0.4 \mathrm{~A}$ to $0.8 \mathrm{~A}$ and measuring the colour change times of the device. Figure 4 shows the relationship between input power and colour change times at an ambient temperature of $22^{\circ} \mathrm{C}$.

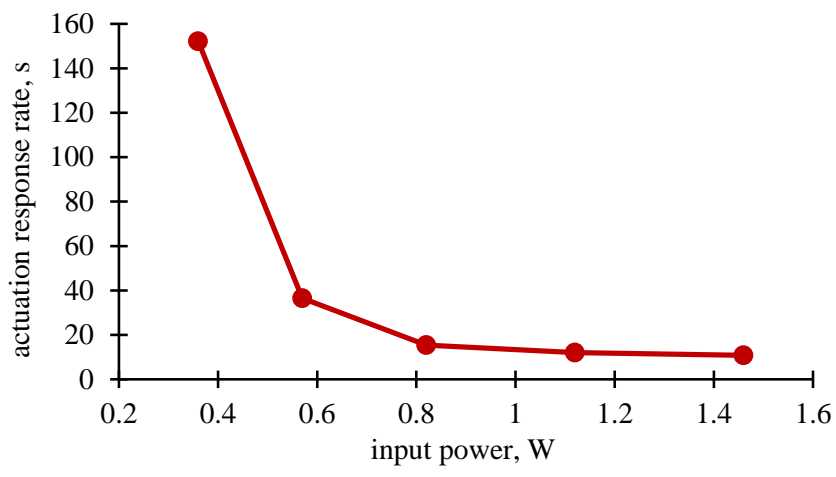

Fig. 4 Relationship between input power and actuation response rate

The results show that an increase in the input power reduces the time it takes to achieve colour change. The response rate of the device improves significantly as input power is increased from $0.36 \mathrm{~W}$ to $0.57 \mathrm{~W}$ however this effect plateaus above this value. It is shown that a fourfold increase in input power of $0.36 \mathrm{~W}$ improves the actuation response of the device by about 14 times to $10.8 \mathrm{~s}$. Creative applications of TC fabrics may require the actuation response to be slow to achieve specific aesthetic effects e.g. shimmering flower [2] whereas functional applications such as non-emissive displays [3] generally require faster response rates than creative applications. The results show 
that the input power to the device can be varied to obtain desired actuation response rates to suit individual applications.

Conclusions: This paper presents a novel all printed approach of fabricating an actively actuated thermochromic device on a polyester cotton substrate for smart fabric applications. The three step novel fabrication method consists of dispenser printing:

- An interface layer on the fabric surface to reduce its surface variation.

- A heater on the interface layer.

- A thermochromic layer over the heater.

This novel approach uses a single fabrication technique, unlike previous devices, and offers greater flexibility and design freedom over current state of the art implementations of thermochromic fabrics.

The fabricated device measuring $30 \times 21.5 \mathrm{~mm}$ is shown to change colour from black to green in $10.8 \mathrm{~s}$ using an input power of $1.46 \mathrm{~W}$. The actuation response rate of the device can be varied to suit the application requirement by varying the input power. The heater in the device can be printed in different patterns to produce bespoke colour change profiles. The device can also be scaled up or down in size to suit the application. Any change in heater pattern, printing substrate and the dimensions of the device will change the power consumption and actuation response of the device.

The reported fabrication process of the TC device can be used to produce flexible colour changing fabric devices for creative and functional applications. Examples of potential applications include dynamic art works, interactive smart fabric systems and non-emissive displays.

Acknowledgments: This work has been supported by the European Commission under the 'Technologies and Scientific Foundations in the field of Creativity' theme in the $7^{\text {th }}$ Framework Programme for Research and Technological Development.

"Data supporting this study are openly available from the University of Southampton repository at: http://dx.doi.org/10.5258/SOTON/389347"

Z. Ahmed, Y.Wei, R.Torah and J.Tudor (Building 59, University of Southampton, Southampton, Hampshire, SO17 1BJ, United Kingdom)

E-mail: Za2g13@soton.ac.uk.

\section{References}

1 Berzowska, J. (2005). Electronic textiles: Wearable computers, reactive fashion, and soft computation. Textile: The Journal of Cloth and Culture, 3(1), 58-75.

2 Berzowska, J. (2004, August). Very slowly animating textiles: shimmering flower. In ACM SIGGRAPH 2004 Sketches (p. 34). ACM.

3 Peiris, R. L., Cheok, A. D., Teh, J. K. S., Fernando, O. N. N., Yingqian, W., Lim, A., ... \& Tharakan, M. (2009, August). Ambikraf: an embedded non-emissive and fast changing wearable display. In ACM SIGGRAPH 2009 Emerging Technologies (p. 1). ACM.

4 Worbin, L. (2010). Designing dynamic textile patterns. Chalmers University of Technology

5 van der Maas, D., Meagher, M., Abegg, C., \& Huang, J. (2009). Thermochromic Information Surfaces. In Proceedings of the International Conference on Education and research in Computer Aided Architectural Design in Europe, eCAADe (Vol. 9, pp. 1-6).

6 Ahmed, Z., Torah, R., \& Tudor, J. (2015, April). Optimisation of a novel direct-write dispenser printer technique for improving printed smart fabric device performance. In Design, Test, Integration and Packaging of MEMS/MOEMS (DTIP), 2015 Symposium on (pp. 1-5). IEEE.

7 Olikrom http://olikrom.com/en/olikrom-products/thermochromic-pigments/ accessed on 03/08/2014 